\title{
LA ENFERMEDAD PULMONAR OBSTRUCTIVA CRÓNICA ESTABLE ASOCIADA A DETERIORO COGNITIVO: POSIBLE FACTOR DE CAUSALIDAD. ESTUDIO PILOTO
}

\author{
STABLE CHRONIC OBSTRUCTIVE PULMONARY DISEASE ASSOCIATED WITH COGNITIVE \\ IMPAIRMENT: POSSIBLE CAUSALITY FACTOR. PILOT STUDY
}

\section{A DOENÇA PULMONAR OBSTRUTIVA CRÔNICA ESTÁVEL ASSOCIADA À DETERIORAÇÃO COGNITIVA: POSSIVVEL FATOR DE CAUSALIDADE. ESTUDO PILOTO}

\author{
Lucía Varela ${ }^{1}$, Facundo Correa ${ }^{1}$, Alexis Cazaux ${ }^{2}$, Agostina Spaccesi $^{2}$, Daniel Antonio Salica ${ }^{2}$, Susana Vanoni ${ }^{1,3}$.
} \begin{abstract}
La enfermedad pulmonar obstructiva crónica conocida por sus siglas EPOC es una condición determinada de manera pricipal por el hábito de fumar cigarrillos. Se sabe que aunque afecta primordial istémical pulmón, casi no hay sistema delorganismo que no sufra algún grado de repercusion. En los ultimos anos se ha puesto empeño en reconocer a la EPOC como una enfermedad gravedad de la EPOC, mayor frecuencia de deterioro cognitivo.
\end{abstract}

\section{Conceptos clave:}

La EPOC es una enfermedad prevalente y se prevé un aumento en los próximos 10 a 15 años a causa del consumo acumulado de tabaco y el envejecimiento poblacional.

Numerosas publicaciones señalan a la EPOC como un factor de riesgo para el desarrollo de trastornos cognitivos.

Es imperativo pesquisar esta condición en los pacientes con EPOC para reconocer la presencia de trastornos cognitivos que empeoren la calidad de vida y/o compliquen la autonomía y en consecuencia la terapéutica de quienes la padecen.

Este trabajo pretende favorecer la conciencia médica sobre la asociación de ambas entidades.

1- Cátedra de Semiología, Hospital San Roque. Facultad de Ciencias Médicas. Universidad Nacional de Córdoba; Argentina. 2- Cátedra de Clínica Médica II - Hospital San Roque Facultad de Ciencias Médicas. Universidad Nacional de Córdoba; Argentina.

3- E-mail de contacto: suvanoni@yahoo.com.ar

\section{Resumen:}

INTRODUCCIÓN: La enfermedad pulmonar obstructiva crónica (EPOC) se define como limitación progresiva y poco reversible al flujo aéreo a consecuencia de una respuesta inflamatoria por inhalación sustancias, en particular humo del tabaco. La enfermedad tiene repercusión sistémica y entre otras, puede aumentar el riesgo de deterioro cognitivo. MATERIALES Y MÉTODOS: Diseño prospectivo, transversal, observacional y analítico. El universo fueron pacientes EPOC que consultaron en dos instituciones de salud desde junio de 2017 a septiembre de 2018. Muestreo no probabilístico, por conveniencia. Se recogieron datos demográficos, de comorbilidades, se aplicó la escalaMCRm de disnea y se valoraron antecedentes de exacerbación de la enfermedad. La gravedad de la EPOC se reconoció por espirometría de acuerdo a GOLD. El deterioro cognitivo fue evaluado mediante el Neuropsi. RESULTADOS: La muestra final incluyó 44 sujetos. De acuerdo a espirometría y GOLD 7 pacientes (15,9\%) clasificaron en el grupo 1 (leve), 19 (43,2\%) en el 2 (moderado), 11 (25 $\%$ ) en el 3 (grave) y $7(15,9 \%)$ en el 4 (muy grave). De los 44 pacientes reclutados, $28(63,6 \%)$ presentaron una función cognitiva normal y $16(36,3 \%)$, algún grado de alteración cognitiva. La memoria fue la función más comprometida. CONCLUSIONES: Dificultades en el reclutamiento impidieron alcanzar el tamaño de muestra esperado. Sin embargo, se observó una tendencia a favor de la asociación. Resulta importante insistir en evitar el tabaquismo pues este parece incrementar el riesgo de deterioro cognitivo que perturba aún más la calidad de vida y dificulta el tratamiento.

Palabras clave: enfermedad pulmonar obstructiva crónica; disfunción cognitiva; tabaquismo.

Abstract:

BACKGRUOUND: Chronic Obstructive Pulmonary Disease (COPD), a preventable and underdiagnosed pathology, can be defined as a progressive and poorly reversible limitation to airflow as a result of a persistent inflammatory response due to inhalation of harmful substances, particularly tobacco smoke. The disease has a systemic impact. Among other conditions, it may increase the risk of cognitive impairment along with its associated consequences. METHODS: Prospective, crosssectional, observational and analytical design. The space were COPD patients who visited in two health institutions from June 2017 to September 2018. Non-probabilistic and convenience sampling. Data were collected on demographics, tobacco consumption, comorbidities (Charlson index), a validated scale for dyspnea (MCRm), the main symptom in COPD and the COPD Assessment Test (CAT) were applied along with a history of exacerbation of the disease. The severity of COPD was evaluated by spirometry according to GOLD guideline. Cognitive impairment was assessed using Neuropsi. OUTCOMES: The final sample consisted of 44 subjects. According to spirometry and GOLD, 7 patients $(15.9 \%)$ classified in group 1 (mild), $19(43.2 \%)$ in group 2 (moderate), $11(25 \%)$ in group 3 (severe) and $7(15.9 \%)$ in group 4 (very serious). Of the 44 patients recruited, $28(63.6 \%)$ had normal cognitive function and $16(36.3 \%)$ had some degree of cognitive impairment. Memory was the most compromised function. CONCLUSION: Difficulties in recruitment impeded reaching the expected sample size. However, a trend was observed favoring the association. It is important to insist on avoiding smoking because it seems to increase the risk of cognitive impairment that further disrupts the quality of life and makes treatment difficult.

Keywords: pulmonary disease, chronic obstructive; cognitive dysfunction; tobacco use disorder.

\section{Resumo}

INTRODUÇÃO: A doença pulmonar obstrutiva crônica (DPOC) é definida como a limitação progressiva e pouco reversivel ao fluxo de ar como resultado de uma resposta inflamatória por substâncias inalatórias, em particular a fumaça do tabaco. A doença tem repercussão sistêmica e, entre outras, pode aumentar o risco de comprometimento cognitivo. MATERIAIS E MÉTODOS: Desenho prospectivo, transversal, observacional e analítico. O universo foram pacientes com DPOC que consultaram em duas instituições de saúde de junho de 2017 a setembro de 2018. Amostragem não probabilística, por conveniência. Os dados demográficos das comorbidades foram coletados, a escala MDRM de dispnéia foi aplicada e uma história de exacerbação da doença foi avaliada. A gravidade da DPOC foi reconhecida pela espirometria, segundo o GOLD. O comprometimento cognitivo foi avaliado polo Neuropsi. RESULTADOS: A amostra final incluiu 44 sujeitos. De acordo com a espirometria e o GOLD, 7 pacientes (15,9\%) foram classificados no grupo 1 (leve), 19 (43,2\%) no 2 (moderado), 11 (25\%) no 3 (grave) e $7(15,9 \%)$ no 4 (muito grave). Dos 44 pacientes recrutados, $28(63,6 \%)$ apresentavam função cognitiva normal e $16(36,3 \%)$, algum grau de comprometimento cognitivo. A memória foi a função mais comprometida. CONCLUSÕES: Dificuldades no recrutamento impediram o tamanho esperado da amostra. No entanto, uma tendência foi observada a favor da associação. É importante insistir em evitar o tabagismo, pois parece aumentar o risco de comprometimento cognitivo que atrapalha ainda mais a qualidade de vida e dificulta o tratamento. 


\section{Introducción}

La enfermedad pulmonar obstructiva crónica (EPOC) es una enfermedad común, prevenible y tratable que se caracteriza por síntomas respiratorios persistentes y limitación del flujo de aire debido a anomalías en las vías aéreas y/o alveolares generalmente causadas por una exposición significativa a partículas o gases nocivos influenciado por factores del huésped incluyendo el anormal desarrollo pulmonar ${ }^{1}$. Se requiere espirometría para hacer el diagnóstico. La presencia de una relación posbroncodilatador $<0,70$ entre el volumen espiratorio forzado en el primer segundo y la capacidad vital forzada (VEF $1 / C V F)$ confirma la EPOC ${ }^{1}$.

La prevalencia global de la enfermedad se estima en 11,7\% en fumadores y exfumadores. En quienes nunca fumaron se establece entre el $3-11 \%$. Su incidencia está en aumento y se encuentra directamente relacionada a prevalencia de tabaquismo. Es la principal causa de morbimortalidad en pacientes que sufren enfermedades crónicas ${ }^{2}$. Provoca unos tres millones de muertes anuales. Con el incremento del consumo de tabaco, la polución y el envejecimiento de la población se calcula que ese número aumentará en los próximos 40 años hasta alcanzar los 5,4 millones de muertes anuales en $2060^{1}$.

En Argentina el estudio EPOC.AR. mostró que la prevalencia es de $14,5 \%$ en personas mayores de 40 años y el subdiagnóstico se ubica alrededor del $75 \%{ }^{3}$.

La espirometría permite clasificar a los pacientes en grados según el deterioro del VEF 1 . Pero existe una significativa heterogeneidad en la presentación clínica y en la progresión de la enfermedad que el $\mathrm{VEF}_{1}$ no describe adecuadamente. Desde hace varios años se intenta caracterizar los fenotipos de la EPOC mediante "un único o una combinación de atributos de la enfermedad que describen las diferencias entre los individuos con EPOC en lo que respecta a los resultados clínicamente significativos: síntomas, exacerbaciones, respuesta a la terapia, tasa de progresión de la enfermedad o muerte" ${ }^{4}$. Se entiende por exacerbación el empeoramiento sostenido del estado del paciente, desde el estado estable y más allá de las variaciones diarias normales, que es agudo en su inicio y requiere un cambio en la medicación habitual ${ }^{4}$.

Los pacientes con EPOC pueden presentar comorbilidades, ya sea independientes o como consecuencia de la repercusión sistémica de la enfermedad o del tabaquismo ${ }^{5}$. Las enfermedades frecuentemente asociadas son cardiopatía isquémica, hipertensión arterial sistémica, osteoporosis, depresión, ansiedad, cáncer de pulmón, asma, infecciones respiratorias, síndrome metabólico, diabetes mellitus, malnutrición y úlcera gastroduodenal ${ }^{1,6}$. Desde hace unos años también se ha relacionado la EPOC con el deterioro cognitivo. La función cognitiva evoca las funciones cerebrales que incluyen el proceso por el cual el individuo percibe, registra, almacena, recupera y usa la información para poder adaptar su comportamiento a nuevas situaciones ${ }^{7}$.
Las personas con EPOC parecen presentar un riesgo aumentado como resultado de diversos factores ${ }^{5}$. La prevalencia oscila del 10 al $61 \%$. Esta variación depende de la muestra del estudio y el método neuropsicológico usado $^{8,9}$.

Existen numerosas pruebas estandarizadas para la evaluación neuropsicológica. Se decidió emplear el Instrumento de Evaluación Neuropsicológica Breve en Español (Neuropsi), desarrollado en Méjico, diseñado para reconocer un amplio espectro de funciones cognitivas incluyendo orientación, atención y activación, memoria, lenguaje, aspectos visoespaciales y visoperceptuales y funciones ejecutivas ${ }^{10}$. Querejeta et al. normativizaron las variantes argentinas del Neuropsi ${ }^{11}$.

El objetivo primario fue conocer la frecuencia de deterioro cognitivo en pacientes con enfermedad pulmonar obstructiva crónica. Los secundarios fueron establecer la relación entre la gravedad de la EPOC mediante GOLD con el grado de deterioro cognitivo evaluado por Neuropsi; reconocer si los pacientes con oxigenoterapia crónica muestran mayor deterioro cognitivo e identificar las funciones cognitivas más deterioradas.

\section{Pacientes y Métodos}

Diseño prospectivo, descriptivo, transversal y analítico. La muestra se conformó con pacientes adultos de ambos sexos con diagnóstico definitivo de EPOC (Guía GOLD 2020) que consultaron para control neumonológico en la Clínica Privada Caraffa y el Hospital Rawson en el período comprendido entre el 01 de junio de 2017 y el 05 de septiembre de 2018. Los otros criterios de inclusión fueron la capacidad de comprender la información y brindar su consentimiento informado. Se excluyó a los pacientes que estuviesen cursando una exacerbación de su enfermedad o padecieran comorbilidades que pudieran modificar la función pulmonar. Asimismo, los pacientes con consumos problemáticos de sustancias o que tuvieran diagnósticos de padecimientos neurocognitivos atribuibles a otro origen. Se recolectaron desde la historia clínica los datos demográficos; antecedentes tóxicos y epidemiológicos y la presencia de comorbilidades de acuerdo con índice de Charlson ${ }^{12}$. Se aplicó la escala clínica de disnea modificada del Medical Research Council $(m M R C)^{13}$ y el COPD Assessment Test $(\mathrm{CAT})^{14}$. La limitación al flujo aéreo (valores del $V E F_{1}$ ) se extrajo desde una espirometría realizada dentro de los 6 meses previos. Con ese resultado se clasificó a los pacientes en grados del 1 al 4 según el valor del $\mathrm{VEF}_{1}$, en consonancia con la Global initiative for chronic Obstructive Lung Disease (GOLD) $2020^{1}$. Con la "herramienta de evaluación" que provee la misma guía y que contempla exacerbaciones, CAT y mMRC se identificaron los grupos $A, B, C$ y $D^{1}$ (Figura $N^{\circ} 1$ ). 


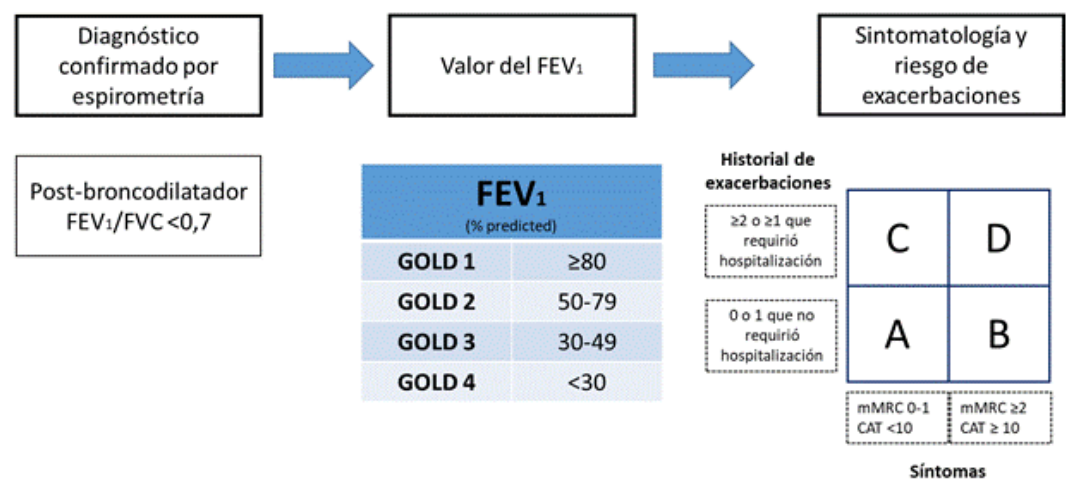

Figura $N^{\circ}$ 1. Clasificación de los pacientes EPOC

Para evaluar las funciones cognitivas se aplicó el "NEUROPSI". Es una prueba constituida por ítems sencillos y cortos, baremado en la población argentina ${ }^{11}$. Permite obtener un puntaje total y un perfil individual de funciones cognitivas. Este perfil señala las habilidades e inhabilidades del sujeto en cada una de las áreas cognitivas evaluadas. De acuerdo con el nivel de escolaridad y edad, los resultados pueden demostrar normalidad o alteraciones leves, moderadas o graves.

Para el análisis estadístico los datos demográficos y de salud no relacionados al deterioro cognitivo, se consideraron como variables dependientes. Se aplicaron tablas de contingencia para calcular el grado de relación entre los resultados del Neuropsi y las categorías de EPOC tanto para la escala combinada como para los grupos según gravedad $^{1}$. Se consideró significativa un valor de $p<$ a 0,05 para un intervalo de confianza del 95\%. Se utilizó el programa estadístico SPSS, versión 23.

La investigación fue aprobada por los Comités de Ética de la Facultad de Ciencias Médicas y del Polo Sanitario (Ministerio de Salud de la Provincia de Córdoba). Los participantes firmaron libre y voluntariamente el consentimiento luego de recibir la información completa y pertinente sobre la investigación.

\section{Resultados}

La muestra final estuvo conformada por 44 sujetos, 25 mujeres $(56,8 \%)$ y 19 hombres $(43,2 \%)$. El rango de edad entre 46 y 88 años, con una media de 63,07 (DE $\pm 8,2$ ). El $95,5 \%(n=42)$ tenía antecedente de tabaquismo. De estos, $93,2 \%(n=41)$ refería tabaquismo activo. Tabaquista actual $19(43,2 \%)$, ex tabaquista (seis meses o más sin fumar), 22 $(50 \%)$ y $1(2,3 \%)$ tabaquista pasivo. Los dos pacientes sin exposición al humo de tabaco refirieron exposición a biomasa uno de ellos y el otro, a la sílice no pudiéndose explicar en este caso, la presencia de EPOC.

En cuanto al índice paquetes/año el mínimo fue de 0 años (aquellos pacientes con antecedente de contaminación ambiental a nivel laboral y exposición al humo de segunda mano) y un máximo de 144 paquete/año. La media fue de $53,93 \pm 34,0$. Se dividió al índice paquetes/año en dos grupos: aquellos con un índice $\geq 40$ en el grupo de fumadores pesados, y grupo de fumadores leves y moderados para pacientes con índice $<40$. En base a esto, se encontró que el $70,5 \%(n=31)$ de los individuos perteneció al primer grupo, mientras que el restante $29,5 \%$ $(n=13)$ al grupo de menor consumo $(n=13)$.

La espirometría permitió dividir a los sujetos según grado de obstrucción al flujo aéreo de acuerdo con la guía GOLD en 7 pacientes $(15,9 \%)$ en el grado 1 (EPOC leve), 19 (43,2\%) en el grado 2 (moderado), 11 (25\%), grado 3 (grave) y 7 $(15,9 \%)$ grado 4 (muy grave). Con respecto al fenotipo de la enfermedad, 50 \% se catalogó como exacerbador. La media de exacerbaciones por año fue de 2,07 (DE $\pm 2,8$ ). La mínima resultó de 0 para una máxima de 12 exacerbaciones en el último año. Como resultado de aplicar la herramienta de evaluación GOLD quedaron 11 pacientes $(25 \%)$ en el grupo A; igual número en el B; $5(11,4 \%)$ en el C y $17(38,6$ $\%$ ) en el grupo D. Al combinar los grupos y los grados se observó que la distribución prevalente fue para grado 1 grupo A con 5 individuos (11,4\%) y $6(13,6 \%)$ pacientes para cada una de las siguientes combinaciones: grupo 2 grado $A$, grupo 2 grado $D$ y grupo 3 grado $D$.

El análisis del deterioro cognitivo mediante el Neuropsi mostró un valor mínimo obtenido en la prueba de 50,5 puntos y un valor máximo de 118 (para un puntaje máximo posible de 130). La media fue de 91,4 (DE $\pm 16,4)$. De los 44 pacientes reclutados, $28(63,6 \%)$ presentaron una función cognitiva normal. El restante $36,3 \% \quad(n=16)$ presentó alteración cognitiva. Esta se dividió en $3(6,8 \%)$ pacientes sobre el total de la muestra, con alteración leve, 10 pacientes $(22,7 \%)$, moderada y otros $3(6,8 \%)$ con alteración grave de las funciones cognitivas. Se encontró mayor porcentaje de hombres con deterioro cognitivo $(47,7$ $\%$ de los hombres vs $28 \%$ de las mujeres), aunque no se pudo establecer una relación significativa entre la presencia de deterioro cognitivo y el sexo $(p=0,186)$ (ver figura $\mathrm{N}^{\circ} 2$ ).

El Neuropsi permitió, mediante el análisis del patrón de errores, identificar las funciones más comprometidas. Para un total de 27 ítems analizados por paciente, la media de ejercicios alterados fue de 8,06 (DE $\pm 3,1$ ). La mínima obtenida fue de 3 y la máxima de 12 ejercicios. Entre los pacientes que presentaron deterioro cognitivo, la memoria resultó ser la función más comprometida. Hasta en un $75 \%$ de la muestra obtuvo alterado alguno de los ejercicios que valoran memoria reciente y hasta un $68,7 \%$ en alguno de los puntos que valoran memoria evocativa. Luego de la memoria, el dominio en el que se encontró mayor alteración fue en las funciones ejecutivas conceptuales (en la prueba de secuenciación), con hasta un $68,7 \%$ de resultados alterados. En tercer orden el dominio más alterado resultó la atención y concentración. 


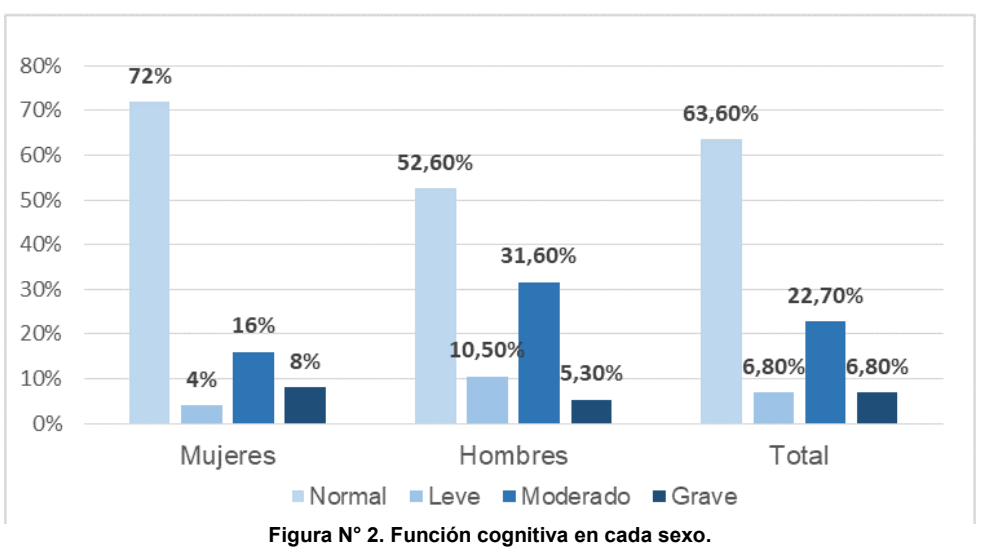

Se aplicaron tablas de contingencia para mostrar la relación entre la gravedad de EPOC y la presencia de deterioro cognitivo. Para evitar la dispersión de la muestra la variable "trastorno cognitivo" obtenida por el Neuropsi se convirtió en categórica: trastorno cognitivo SI (Neuropsi alterado) y NO (Neuropsi normal). Para la variable "gravedad de la EPOC", se crearon dos clases: clase 1 (grupos EPOC leve y moderado) y clase 2 (Grupos EPOC grave y muy grave de la clasificación GOLD). Se obtuvo una $p$ no significativa $(p=0,118)$. Los resultados arrojaron que 26 de los 44 pacientes categorizaron como clase $1(59,1 \%)$ con $19(73,1$ $\%$ del total de esta clase) presentaron función cognitiva normal. En cambio, de los 18 pacientes de la clase 2, solo la mitad (9 sujetos) presentó función cognitiva normal. (ver tabla $\left.N^{\circ} 1\right)$.

Tabla $\mathrm{N}^{\circ}$ 1: Relación entre gravedad de EPOC y deterioro cognitivo

\begin{tabular}{|c|c|c|c|c|c|c|c|c|}
\hline & & & \multicolumn{5}{|c|}{ Función cognitiva } & \multirow[b]{2}{*}{ Total } \\
\hline & & & Normal & Alt. Leve & $\begin{array}{c}\text { Alt. } \\
\text { Moderada }\end{array}$ & Alt. Grave & $\begin{array}{c}\text { Total } \\
\text { alteración } \\
\text { cognitiva }\end{array}$ & \\
\hline \multirow{6}{*}{$\begin{array}{l}\mathrm{VEF}_{1} \\
\text { Clasificación } \\
\text { por clases }\end{array}$} & \multirow{3}{*}{$\begin{array}{l}\text { EPOC leve y } \\
\text { moderado }\end{array}$} & Recuento & 19 & 2 & 4 & 1 & 7 & 26 \\
\hline & & $\begin{array}{l}\text { Porcentaje } \\
\text { dentro del } \\
\text { grupo } 1\end{array}$ & $73,10 \%$ & $7,70 \%$ & $15,40 \%$ & $3,80 \%$ & $26,90 \%$ & $100,00 \%$ \\
\hline & & $\begin{array}{l}\text { Porcentaje } \\
\text { del total }\end{array}$ & $43,20 \%$ & $4,50 \%$ & $9,10 \%$ & $2,30 \%$ & $15,90 \%$ & $59,10 \%$ \\
\hline & \multirow{3}{*}{$\begin{array}{l}\text { EPOC grave } \\
\text { y muy grave }\end{array}$} & Recuento & 9 & 1 & 6 & 2 & 9 & 18 \\
\hline & & $\begin{array}{l}\text { Porcentaje } \\
\text { dentro del } \\
\text { grupo } 2\end{array}$ & $50,00 \%$ & $5,60 \%$ & $33,30 \%$ & $11,10 \%$ & $50,00 \%$ & $100,00 \%$ \\
\hline & & $\begin{array}{l}\text { Porcentaje } \\
\text { del total }\end{array}$ & $20,50 \%$ & $2,30 \%$ & $13,60 \%$ & $4,50 \%$ & $20,50 \%$ & $40,90 \%$ \\
\hline \multirow{2}{*}{\multicolumn{2}{|c|}{ Total }} & Recuento & 28 & 3 & 10 & 3 & 16 & 44 \\
\hline & & $\begin{array}{l}\text { Porcentaje } \\
\text { del total }\end{array}$ & $63,60 \%$ & $6,80 \%$ & $22,70 \%$ & $6,80 \%$ & $36,40 \%$ & $100,00 \%$ \\
\hline
\end{tabular}

En cuanto a la relación entre deterioro cognitivo y el uso de oxigenoterapia crónica, no se obtuvo diferencia estadísticamente significativa $(p=0,572)$. Empleaba oxígeno el $20,5 \%(n=9)$ de los cuales 5 tenían función cognitiva normal. Los restantes 35 pacientes, 65,7\% $(n=23)$ también mostraron función cognitiva normal.

\section{Discusión}

Las principales debilidades de esta investigación se relacionan con el número de pacientes incluidos, determinado por el carácter de estudio piloto, y la técnica de muestreo no probabilístico por conveniencia. Hecha esta salvedad, el análisis mostró algunas evidencias similares a lo señalado por otros autores con relación a EPOC y deterioro cognitivo que se analizarán de manera específica.
Esta muestra incluyó solo pacientes con diagnóstico definido de EPOC, por lo cual no puede analizarse prevalencia de la enfermedad. Con relación al sexo, predominaron ligeramente las mujeres $(56,8 \%)$ posiblemente porque el reclutamiento fue en instituciones de salud y las mujeres en general, muestran un mayor apego a los controles en salud. Hasta hace algunos años la EPOC era más frecuente en hombres. De hecho, el estudio del grupo Platino publicado en 2013 , demostró que el $52,3 \%$ eran hombres ${ }^{15}$.

En este estudio se objetivó al igual que en otras publicaciones, que la mayoría de los pacientes son tabaquistas actuales o pasados $(93,2 \%$ de la muestra, $\mathrm{n}=41$ ) y que además un porcentaje considerable continúa fumando a pesar del diagnóstico. Se estima que el humo de tabaco es la causa más común de EPOC y se presume que solo el $20 \%$ de las personas que la padecen nunca fumó ${ }^{15}$. En cambio, la muestra analizada arrojó un porcentaje 
menor, solo el 6,8 \% carecía de esa exposición. Pero debe considerarse la característica de estudio piloto del presente trabajo.

Con relación a los resultados de la espirometría esta casuística no amerita comparación con el estudio EPOC.AR porque la actual incluyó pacientes con diagnóstico definitivo de EPOC y el argentino fue un estudio poblacional para reconocer prevalencia de la enfermedad en el país ${ }^{3}$. En cambio, la gravedad de la EPOC encontrada se correlaciona muy bien con un ensayo publicado en Holanda donde sobre 183 pacientes con EPOC, 15,8 \% clasificaron como EPOC leve; $38,3 \%$ como moderado; $33,3 \%$ grave y $12,6 \%$ como muy grave ${ }^{16}$.

El análisis de esta casuística identificó que un 36,4 \% de los pacientes presentaba algún grado de deterioro cognitivo. Otros estudios han reportado una frecuencia del 10 al $61 \%$ de deterioro enlazado a EPOC dependiendo de la población estudiada y de la prueba neuropsicológica aplicada ${ }^{5,16}$. Una revisión sistemática señaló que el deterioro se asocia a la severidad de la EPOC y en esta muestra solo algo más del $15 \%$ padecía enfermedad pulmonar muy grave ${ }^{17}$. La prevalencia en los pacientes de esta casuística se encuentra dentro de la frecuencia reportada y es mayor a lo esperado para la población general ${ }^{17}$. Para ese mismo grupo Torres Sánchez et. al. identificaron en la revisión publicada una frecuencia del 10 al $20 \%$ en adultos $\geq 65$ años $^{18}$. El dato impacta más aún si se observa que la edad de la población es comparable a la esta casuística.

Un elemento interesante para tener en cuenta es que muchos de los estudios realizados sobre el tema, utilizan test de tamizaje para alteraciones cognitivas que no tienen en cuenta la edad del paciente ni la escolaridad. Como ya se mencionó, esta es una de las fortalezas del Neuropsi aplicado en este trabajo, además de la validación local ${ }^{11}$.

Se ha demostrado que el deterioro cognitivo en pacientes con EPOC puede afectar solo algunos dominios o de manera global. Además, ha sido reportado el empeoramiento del deterioro según el tiempo de evolución de la EPOC ${ }^{19}$. El análisis de los dominios en esta muestra demostró que la memoria resultó el ítem alterado con mayor frecuencia. Las dos terceras partes mostraron alteración de la memoria evocativa y/o reciente. La revisión de Torres Sánchez et al. señaló también a la memoria como uno de los dominios más afectados. Entre las otras funciones cognitivas alteradas con mayor frecuencia le siguieron las funciones ejecutivas conceptuales, atención y concentración, comprensión, lectura y orientación temporal $^{18}$

Campman et al., encontraron que la velocidad de procesamiento fue el dominio que en más pacientes se encontró alterado $^{20}$. Cleutjens et al. (2017), en su investigación hallaron que la prevalencia de deterioro cognitivo fue más elevada en los dominios de planeamiento y flexibilidad cognitiva ${ }^{21}$. Sin embargo, también se han visto afectados en pacientes EPOC la memoria, aprendizaje, atención, concentración, fluidez verbal, velocidad de procesamiento, coordinación, inteligencia y flexibilidad cognitiva $^{16}$.

Además, otro dato de interés arrojado por Campman et. al., en su estudio, fue demostrar que los pacientes que tenían deterioro cognitivo presentaban una $\mathrm{PaO} 2$ más baja y/o una $\mathrm{PaCO} 2$ más elevada ${ }^{20}$. En el trabajo presentado hasta un $44,4 \%$ de los pacientes con oxigenoterapia presentó deterioro cognitivo, valor algo superior al $34,4 \%$ encontrado en pacientes sin requerimientos de oxígeno. En cambio, la revisión de Torres Sánchez et al no fue tan categórica para relacionar hipoxemia con deterioro ${ }^{19}$.

Por su parte, la relación entre la disfunción cognitiva y la gravedad de la obstrucción al flujo aéreo aún tiene que ser establecida. En general, no parece presentar una asociación lineal, es decir, a EPOC más grave, más deterioro cognitivo. $Y$ existen publicaciones a favor y en contra de esa relación ${ }^{16-17,21}$. En la serie de pacientes analizados, si bien se logró ver una tendencia a presentar de manera más frecuente deterioro cognitivo según avanza la gravedad de la enfermedad, no se pudo establecer una relación significativa.

En esta muestra no se identificó el fenotipo exacerbador de los pacientes con la presencia de mayor deterioro cognitivo, excepto cuando se analizó la muestra separada según el sexo. Las mujeres con fenotipo exacerbador mostraron disfunción cognitiva más frecuente $(p=0,035)$. Sin embargo, el avance en la limitación al flujo aéreo, la hipoxemia, la hipercapnia y las exacerbaciones podrían inducir un deterioro cognitivo más temprano y grave en pacientes EPOC $^{16}$.

\section{Conclusión}

El trabajo demostró una frecuencia elevada de deterioro cognitivo en los pacientes con EPOC que se ubica por encima de la esperada para la población en general. No logró establecerse una clara relación entre la severidad de la EPOC y el grado de deterioro cognitivo. La oxígenoterapia crónica no pudo identificarse como una condición adicional en favor de la disfunción mental de los pacientes. Las funciones o dominios más comprometidos fueron la memoria, las funciones ejecutivas conceptuales y, en tercer lugar, la atención y concentración. La principal debilidad de las conclusiones es la condición de estudio piloto. Adicionalmente, los resultados sustentan la necesidad de ampliar las investigaciones para reconocer la magnitud de la asociación EPOC y estado mental y de efectuar una evaluación cognitiva estructurada de estos pacientes. La repercusión sobre la función cognitiva afecta la calidad de vida y complica aún más la limitada independencia de los pacientes, marcando un riesgo al momento de prescribir tratamiento farmacológico o rehabilitación.

\section{Agradecimiento:}

Agradecimiento: al Dr. Arnaldo Pedro Mangeaud por su orientación inicial en el análisis estadístico.

\section{Limitaciones de responsabilidad:}

Todos los autores somos responsables de los contenidos vertidos. Hemos participado con las siguientes contribuciones. La Dra. Susana Vanoni, coordinadora del equipo, propuso el tema central de la investigación y junto a la médica Lucía Varela plantearon la hipótesis y los objetivos y el diseño inicial. El Dr. Daniel Salica y el médico Alexis Casaux realizaron aportes específicos sobre selección de pacientes y metodología. Casaux y Vanoni tuvieron a su cargo la selección de pacientes EPOC y accedeer a los datos de la historia clínica (pacientes propios). Lucía Varela y Agostina Spaccesi reclutaron los participantes, obtuvieron el consentimiento informado y aplicaron el Neuropsi bajo la dirección del médico psiquiatra Facundo Correa. Correa tuvo a su cargo la revisión de los resultados del Neuropsi. Varela y Vanoni realizaron el procesamiento estadístico de los datos y la descripción de los resultados. Varela escribió la introducción, pacientes y métodos, resultados, discusión y conclusiones bajo la directa supervisión de la Dra. Vanoni. Todos los coautores revisaron el manuscrito final y realizaron aportes y sugerencias. 


\section{Fuentes de apoyo:}

Los autores no hemos recibido apoyo económico ni subsidios para la realización de este trabajo.

\section{Originalidad:}

Este artículo es original y no ha sido enviado para su publicación a otro medio de difusión científica en forma completa ni parcialmente.

\section{Cesión de derechos:}

Los participantes de este trabajo ceden el derecho de autor a la Universidad Nacional de Córdoba para publicar en la Revista de la Facultad de Ciencias Médicas y realizar las traducciones necesarias al idioma inglés.

\section{Conflicto de interés:}

Los autores no tenemos conflictos de interés para declarar con respecto al presente manuscrito.

\section{Bibliografía}

1.Global Initiative for Chronic Obstructive Lung Disease. Global Strategy for the diagnosis, management, and the prevention of Chronic Obstructive Pulmonary Disease. Report (2020). Disponible en: https://goldcopd.org/wpcontent/uploads/2019/12/GOLD-2020-FINAL-ver1.203Dec19 WMV.pdf

2.Schou L, Østergaard B, Rasmussen LS, Rydahl-Hansen S, Phanareth K. Cognitive dysfunction in patients with chronic obstructive pulmonary disease a systematic review. Respir Med. 2012 Aug;106(8):1071-81. doi: 10.1016/j.rmed.2012.03.013.

3.Echazarreta AL, Arias SJ, Del Olmo R, Giugno ER, Colodenco FD, Arce SC, Bossio JC, Armando G, Soriano JB; Grupo de estudio EPOC.AR. Prevalencia de enfermedad pulmonar obstructiva crónica en 6 aglomerados urbanos de Argentina: el estudio EPOC.AR. Arch Bronconeumol. 2018 May;54(5):260-269. English, Spanish. doi: 10.1016/j.arbres.2017.09.018.

4. Han MK, Agusti A, Calverley PM, Celli BR, Criner G, Curtis JL, Fabbri LM, Goldin JG, Jones PW, Macnee W, Make BJ, Rabe KF, Rennard SI, Sciurba FC, Silverman EK, Vestbo J, Washko GR, Wouters EF, Martinez FJ. Chronic obstructive pulmonary disease phenotypes: the future of COPD. Am J Respir Crit Care Med. 2010 Sep 1;182(5):598-604. doi: 10.1164/rccm.200912-1843CC.

5. Dodd JW. Lung disease as a determinant of cognitive decline and dementia. Alzheimers Res Ther. 2015 Mar 21;7(1):32. doi: 10.1186/s13195-015-0116-3.

6. Cavaillès A, Brinchault-Rabin G, Dixmier A, Goupil F, Gut-Gobert C, Marchand-Adam S, Meurice JC, Morel H, Person-Tacnet C, Leroyer C, Diot P. Comorbidities of COPD. Eur Respir Rev. 2013 Dec;22(130):454-75. doi: 10.1183/09059180.00008612.

7.Cleutjens FA, Janssen DJ, Ponds RW, Dijkstra JB, Wouters EF. COgnitive-pulmonary disease. Biomed Res Int. 2014;2014:697825. doi: 10.1155/2014/697825.

8. Pérez Martínez VT. El deterioro cognitivo: una mirada previsora. Rev Cubana Med Gen Integr. 2005;21(1-2).

9.Andrianopoulos V, Gloeckl R, Vogiatzis I, Kenn K. Cognitive impairment in COPD: should cognitive evaluation be part of respiratory assessment? Breathe (Sheff). 2017 Mar;13(1):e1-e9. doi: 10.1183/20734735.001417.

10. Ostrosky-Solís, F. y Lozano Gutiérrez, A. Factores Socioculturales en la valoración neuropsicológica. Revista Argentina de Ciencias del Comportamiento, 2012;4(2):43-50. doi: 10.32348/1852.4206.v4.n2.5173.

11. Querejeta, A, Farías Sarquís $Y$, Moreno MA, Crostelli AL, Stecco JI, Venier A, Godoy JC. Pilatti A. Test Neuropsi: Normas según edad y nivel de instrucción para Argentina. Cuadernos de Neuropsicología. 2012;6(2):48-58. doi: 10.7714/cnps/ 6.2.204.

12. Brusselaers N, Lagergren J. The Charlson Comorbidity Index in Registry-based Research. Methods Inf Med. 2017;56(5):401-406. doi: 10.3414/ME17-01-0051

13. Perez T, Burgel PR, Paillasseur JL, Caillaud D, Deslée G, Chanez $P$, Roche N; INITIATIVES BPCO Scientific Committee. Modified Medical Research Council scale vs Baseline Dyspnea Index to evaluate dyspnea in chronic obstructive pulmonary disease. Int J Chron Obstruct Pulmon Dis. 2015 Aug 18;10:166372. doi: 10.2147/COPD.S82408.

14. Jones PW, Harding G, Berry P, Wiklund I, Chen WH, Kline Leidy $N$. Development and first validation of the COPD Assessment Test. Eur Respir J. 2009 Sep;34(3):648-54. doi: 10.1183/09031936.00102509.

15. López Varela MV, Montes de Oca M, Halbert R, Muiño A, Tálamo C, Pérez-Padilla R, Jardim JR, Valdivia G, Pertuzé J, Menezes AM; PLATINO team. Comorbilidades y estado de salud en individuos con y sin EPOC en 5 ciudades de América Latina: Estudio PLATINO. [Comorbidities and health status in individuals with and without COPD in five Latin American cities: the PLATINO study]. Arch Bronconeumol. 2013 Nov;49(11):468-74. English, Spanish. doi: 10.1016/j.arbres.2013.05.003.

16. Cleutjens FAHM, Spruit MA, Ponds RWHM, Vanfleteren LEGW, Franssen FME, Gijsen C, Dijkstra JB, Wouters EFM, Janssen $D J A$. Cognitive impairment and clinical characteristics in patients with chronic obstructive pulmonary disease. Chron Respir Dis. 2018 May;15(2):91-102. doi: 10.1177/1479972317709651.

17. Yazar EE, Aydin S, Gunluoglu G, Kamat S, Gungen AC, Yildiz $P$. Clinical effects of cognitive impairment in patients with chronic obstructive pulmonary disease. Chron Respir Dis. 2018 Aug;15(3):306-314. doi: 10.1177/1479972317743757.

18. Torres-Sánchez I, Rodríguez-Alzueta E, Cabrera-Martos I, López-Torres I, Moreno-Ramírez MP, Valenza MC. Cognitive impairment in COPD: a systematic review. J Bras Pneumol. 2015 Mar-Apr;41(2):182-90. doi: 10.1590/S1806-37132015000004424.

19. Dodd JW, Getov SV, Jones PW. Cognitive function in COPD. Eur Respir J. 2010 Apr;35(4):913-22. doi: 10.1183/09031936.00125109. Erratum in: Eur Respir J. 2010 Jul;36(1):223.

20. Campman C, van Ranst D, Meijer JW, Sitskoorn M. Computerized screening for cognitive impairment in patients with COPD. Int J Chron Obstruct Pulmon Dis. 2017 Oct 19;12:30753083. doi: 10.2147/COPD.S142871.

21. Cleutjens FA, Franssen FM, Spruit MA, Vanfleteren LE, Gijsen C, Dijkstra JB, Ponds RW, Wouters EF, Janssen DJ. Domainspecific cognitive impairment in patients with COPD and control subjects. Int J Chron Obstruct Pulmon Dis. 2016 Dec 19;12:1-11. doi: 10.2147/COPD.S119633. 